Review Article

\title{
LASER - A RAY OF HOPE IN PERIODONTICS - A REVIEW ARTICLE
}

\author{
Amitha Ramesh ${ }^{1}$, Rahul Bhandary ${ }^{2}$, Biju Thomas $^{3}$ \& Sheehan R. Dsouza ${ }^{4}$ \\ ${ }^{1,2}$ Professors, ${ }^{3}$ Professor \& HOD, ${ }^{4}$ P. G. Student, Department of Periodontics,
}

A.B. Shetty Memorial Institute of Dental Sciences, Nitte University, Mangalore - 575 018, Karnataka, India.

\section{Correspondence : \\ Sheehan R. Dsouza}

P.G. Student, Department of Periodontics, A.B. Shetty Memorial Institute of Dental Sciences, Nitte University

Mangalore - 575 018, Karnataka, India.

Mobile : +91 9964668191 E-mail : sheehan_dsouza999@yahoo.com

\section{Abstract:}

Lasers have revolutionised dental treatment since three and a half decades of the twentieth century. Theodore maiman in 1960 invented the ruby laser, since then laser is one of the most captivating technologies in dental practice. Lasers have been used in initial periodontal therapy, surgery, and also in implant treatment. Further research is necessary so that laser can become a part of the dental armamentarium. This paper gives an insight to laser in Periodontics

Keywords : Laser, periodontics, periodontology.

\section{Introduction :}

In the past 100 years there has been extensive development of the mechanical cutting devices used in dentistry. However, while considerable progress has been made in this area of mechanical cutting, dental patients are still afraid of the noise and vibration produced by the mechanical action of the air turbine and ultrasonic scalers. From the end of the $20^{\text {th }}$ century until now, there has been a continuous upsurge in the development of laser-based dental devices based on photomechanical interactions.

The dental lasers of today have benefited from decades of laser research and have their basis in certain theories from the field of quantum mechanics, initially formulated during the early 1900s by Danish physicist Bohr, among others. Nearly 40 years later, American physicist Townes first amplified microwave frequencies by the stimulated emission process, and the acronym MASER (Microwave Amplification by Stimulated Emission of Radiation) came

\begin{tabular}{|c|}
\hline Access this article online \\
\hline Quick Response Code \\
\hline
\end{tabular}
into use. In 1958, Schawlow and Townes discussed extending the maser principle to the optical portion of $t h e$ electromagnetic field; hence, LASER (Light Amplification by Stimulated
Emission of Radiation) was invented.

Lasers designed for surgery deliver concentrated and controllable energy to tissue. For a laser to have biological effect, the energy must be absorbed. The degree of absorption in tissue will vary as a function of the wavelength and optical characteristics of the target tissue. If the peak emission of the laser matches the absorption spectrum of one or more components of the target tissue, a predictable and specific interactive effect will occur. Since tissues all have more than one component, the overall effect will be a combination of the effects, on each tissue component.

\section{Laser Effects on Tissue}

The light energy from a laser can have four different interactions with the target tissue, and these interactions will depend on the optical properties of that tissue.

When radiant energy is absorbed by tissue, four basic types of interactions or responses may occur.

1. Photochemical interaction.

2. Photo thermal interaction

3. Photo mechanical interaction

4. Photo electrical interaction

\section{Photo chemical interaction include}

Bio-stimulation, which describes the stimulatory effects of 
laser light on biochemical and molecular processes that normally occur in tissues such as healing and repair.

\section{Photo thermal interactions include}

Photo ablation, or the removal of tissue by vaporization and superheating of tissue fluids, coagulation and hemostasis.

\section{Photomechanical interaction include}

Photo-disruption or photo-disassociation, which is the breaking apart of structures by laser light.

\section{Photoelectrical interactions include}

Photo plasmolysis which describes how tissue is removed through the formation of electrically charged ions and particles that exist in a semi-gasseous high energy state.

\section{Applications in Dentistry}

I. Intra oral soft tissue surgery
a. Ablating, incising, excising, coagulating
b. Laser-assisted uvulophlatoplasty
c. Treatment of pathologic condition.
d. Sulcular debridement

II. Hard tissue application
a. Caries removal, inhibition, detection
b. Cavity preparation
c. Surface modification
d. Tooth bleaching
e. Calculus removal
f. Bone ablation and cartilage reshaping
g. Dentin desensitization
h. Analgesia

III. Dental Materials

1. Composite curing, bracket bonding

2. Alloy welding

\section{Endodontics}

V. Other application
a. Laser diagnostic
b. Instrument sterilization
c. Holography
d. Bio-stimulation

\section{Uses of Lasers In Periodontics}

Treatment of Dentine Hypersensitivity With Lasers:

Dentine hypersensitivity is characterized by short, sharp, pain arising from exposed dentine in response to stimuli typically thermal evaporative tactile, osmotic or chemical and which can not be ascribed to any other form of dental defect or pathology.

The lasers used for the treatment of dentine hypersensitivity are divided in to two groups: Low level lasers like He-Ne, GaAlAs, and Middle output lasers like Nd: YAG and $\mathrm{CO}_{2}$ lasers. The mechanism of laser effects on dentine hypersensitivity is thought to be the laser induced occlusion or narrowing of dentinal tubules (Lan \& Liu 1995), as well as direct nerve analgesia, via pulpal nerve system. It has been hypothesized that the laser energy interferes with the sodium pump mechanism changes the cell membrane permeability and / or temporarily alters the endings of the sensory axons.

\section{Laser Deepithelization For Enhanced GTR:}

Successful treatment of periodontal defects to obtain new attachment continues to represent a serious therapeutic challenge for predictable result in periodontics.

Historically, many techniques have been tried to retard epithelial down growth. The $\mathrm{CO}_{2}$ laser creates a rather unique wound in the gingival tissue. It is not a burn, rather an instantaneous vaporization of the intercellular fluid and a resulted disintegration of the cell structure.

The laser wound on skin and gingiva causes a delay in reepithlization because of factors such as reduced inflammatory response and less wound contraction. Rossman et al did a 28 day study on monkeys and evaluated the correlation of interproximal defects using $\mathrm{CO}_{2}$ treated sites with control sites; the study indicated a greater amount of connective tissue rather then epithelial attachment.

\section{Depigmentation With Laser:}

Gingival and cutaneous melanin pigmentation is often a source of an aesthetic problem. The intensity and extent of 
pigmentation varies widely among individuals. Various methods suitable for the removal of pigmentation from the gingiva have been described. Among them are cryotherapy, gingivectomy and argon laser irradiation. In addition several lasers are used for ablation of cutaneous pigmented lesions and oral lesions, among them are ruby, dyed pulsed, $\mathrm{Nd}: \mathrm{YAG}, \mathrm{CO}_{2}$ and eximer laser.

\section{Peri Implant Care with Laser:}

Implant maintenance has included methods that mimic the care of periodontium after periodontic manipulations or surgery. With objective in mind of avoiding deleterious alteration of the surface of implant during routine maintenance procedures or in the treatment of failing implants, the Nd:YAG laser was studies a possible modality for detoxifying, debriding and sterilizing the surface of HAcoated and titanium plasma - sprayed (TPS) implants.

\section{Soft Tissue Applications:}

Traditional use of lasers for soft tissue ablation includes gingivectomy, frenectomy, removal of muco-cutaneous lesions (both benign and malignant) and gingival sculpting techniques associated with implant therapy and mucocutaneous surgery.

\section{Laser Bleaching}

The objective of laser bleaching is to achieve the ultimate power bleaching process using the most efficient energy source, while avoiding any adverse effects. Using the 488$\mathrm{nm}$ argon laser as an energy source to excite the hydrogen peroxide molecule offers more advantages than other heating instruments. Argon lasers emit fairly short wavelengths $(488 \mathrm{~nm})$ with higher-energy photons; conversely, plasma-arc lamps, halogen lamps, and other heat lamps emit short wavelengths as well as longer invisible infrared thermal wavelengths (750 $\mathrm{nm}$ to $1 \mathrm{~mm}$ ) with lower-energy photons and predictable high thermal character. This high thermal energy can create unfavorable pulpal responses.

The argon laser rapidly excites the already unstable and reactive hydrogen peroxide molecule; the energy then is absorbed into all intramolecular and intermolecular bonds and reaches eigenstate vibrations. ${ }^{4}$ The hydrogen peroxide molecule falls apart into different, extremely reactive ionic fragments that swiftly combine with the chromophilic structure of the organic molecules, altering them and producing simpler chemical chains. The result is a visually whitened tooth surface.

\section{Esthetic gingival procedures}

Lasers can be applied in esthetic procedures such as recontouring or reshaping of gingiva and in crown Lengthening. With the use of some lasers, the depth and amount of soft tissue ablation is more precisely and delicately controlled than with mechanical instruments. In particular, the Er:YAG laser is very safe and useful for esthetic periodontal soft tissue management because this laser is capable of precisely ablating soft tissues using various fine contact tips, and the wound healing is fast and favorable owing to the minimal thermal alteration of the treated surface.

\section{Nonsurgical pocket therapy \\ Conventional root debridement}

In periodontal pockets the exposed root surfaces are contaminated with an accumulation of plaque and calculus, as well as infiltration of bacterial endotoxins into the cementum. Usually, in the Initial phase of periodontal therapy, debridement of the diseased root surface is nonsurgically treated by mechanical scaling and root planing, primarily by using manual or power-driven instruments.

However, complete removal of bacterial deposits and their toxins from the root surface within the periodontal packets is not always achieved with only the use of conventional mechanical therapy. In addition, access to areas such as furcations and grooves is limited owing to the complicated root anatomy. Further conventional mechanical debridement using curettes is still technically demanding and time-consuming, and power scalers sometimes cause discomfort and stress in patients as a result of noise and vibration. Recently, the benefits of lasers, such as ablation, bactericidal and detoxification effects, as well as photobiomodification, have been reported to be useful for 
periodontal pocket treatment, and the application of lasers has been suggested as an adjunctive or alternative tool to 'conventional periodontal mechanical therapy.

\section{Removal of subgingival calculus}

The $\mathrm{CO}_{2}$ laser cannot be used for calculus removal because this laser readily causes melting and caronization on the dental calculus. The Nd:YAG laser is also basically ineffective for calculus removal when the clinically suitable energy is employed, Unlike these lasers, the Er:YAG laser is capable of easily removing subgingival calculus without a major thermal change of the root surface. The level of calculus removal by this laser is similar to ultrasonic scaling, and the depth of cementum ablation has been reported generally to be $15-30 \mu \mathrm{m}$ when the contact tip is applied obliquely to the root surface. Furthermore, Er:YAG laser treatment in vivo might provide selective subgingival calculus removal to a level equivalent to that provided by scaling and root planing. Recently, a similar performance for calculus removal has been reported with the $\mathrm{Er}, \mathrm{Cr}$ :YSGG laser. However, a lower degree of calculus removal with the Er:YAG laser than with scaling and root planing has also been noted in another in vivo study.

\section{Root surface alterations :}

The $\mathrm{CO}_{2}$ laser readily carbonizes the root cementun, and cyan-derived toxic products, such a cyanamide and cyanate ions, have been clearly detected on the carbonized layer by chemical analysis using Fourier transform infrared spectroscopy. The residual char layer has been demonstrated to inhibit periodontal soft tissue attachment in vivo, and thus focused $\mathrm{CO}_{2}$ laser irradiation is contraindicated for root surface treatment.

\section{Bactericidal and detoxification effects}

Conventional methods for the treatment of periodontal disease are not completely effective in eliminating all types of bacteria. Although systemic and local administration of antibiotics into periodontal pockets is occasionallyeffective for disinfection, the frequent usage of antibiotics bears the potential risk of producing various resistant microorganisms. These limitations have led to a shift in emphasis from a purely mechanical approach to the use of novel technical modalities having additional bactericidal effects, such as lasers.

\section{Periodontal pocket treatment}

One of the possible advantages of laser treatment of periodontal pockets is the debridement of the soft tissue wall. Conventional mechanical tools are not effective for the complete curettage of the soft tissue. Gold \& Vilardi reported the safe application of the Nd:YAG laser (1.25 and $1.75 \mathrm{~W}, 20 \mathrm{~Hz}$ ) for removal of the pocket-lining epithelium in periodontal pockets without causing necrosis or carbonization of the underlying connective tissue in vivo. Recently, use of an Nd:YAG laser in a laser-assisted new attachment procedure has been advocated to remove the diseased soft tissue on the inner gingival surface of periodontal pockets,(Food and Drug Administration $510 \mathrm{k}$ clearance k030290). Quite recently, a case series by Yukna et al. reported that the laser- assisted new attachment procedure could be associated with cementum-mediated new connective tissue attachment and apparent periodontal regeneration on previously diseased root surface in humans.

\section{Surgical pocket therapy}

In order for a periodontal surgical procedure to be successful with optimal tissue regeneration, it is necessary for the root surface and bone defect to be completely debrided and decontaminated. Laser application is effective in debriding areas of limited accessibility, such, as deep intrabony defects and furcation areas where mechanical instruments cannot eliminate microbiological etiologic factors. Laser irradiation can facilitate complete debridement of the defect as a result of its ablation effect as well as improved accessibility when there is contact of the tip of the laser.

\section{The Advantages and Disadvantages of Laser Application In Periodontal Therapy}

\section{Advantages:}

Because of the photo-physical characteristics of lasers, laser irradiation exhibits strong ablation, hemostasis, detoxification and bactericidal effects on the human body. 
These effects could be beneficial during periodontal treatment, especially for the fine cutting of soft tissue as well as in the debridement of diseased tissue. This, in periodontal therapy, laser treatment may serve as an alternative or adjunctive therapy to mechanical approaches. Previously introduced laser systems showed strong side effects, causing melting, cracking and carbonization of hard tissues, such as root and bone. The recently developed Er: YAG and Er,Cr:YSGG lasers, however, can ablate both soft and hard tissues safely with water irrigation and are applicable to periodontal treatments such as scaling, debridement and bone surgery, and have minimal thermal effect. Thus, the erbium laser group has shown promise as a laser system for periodontal treatment approaches on hard tissues. ${ }^{9}$

\section{Disadvantages:}

First, the high financial cost of a laser apparatus is a significant barrier for laser utilization by periodontal practitioners. Second, each laser has different characteristics because of their different wavelengths. Thus, laser users should know the fundamental characteristics of each laser. However, only a few academic institutions provide proper and systematic education of the use of lasers in dentistry. For this reason, it is difficult for the users to learn all aspects of the techniques and References :

1. An Overview of Lasers in Dentistry; CAcademy of Laser Dentistry 2008 Pg 1-18.

2. The Use of a Diode Laser in Periodontics: Lambda Scientifica.

3. AAP-Commissioned Review,Lasers in Periodontics: A Review of the Literature Charles M. Cobb* J Periodontol 2006;77: 545-564.

4. Lasers and light amplification in dentistry, The role of lasers in cosmetic dentistry:Grace Sun, DDS.

5. LANAP ${ }^{\mathrm{TM}}$ and Lasers in Dentistry: On the Frontier of 21st Century Periodontal Therapy,: Dr.Charles Braga,January 2009.

6. Photomedicine and Laser Surgery, George E. Romanos, Marco Henze, Sarah Banihashemi, Hamid R. Parsanejad, Jürge Winckler, GeorgeHubertus Nentwig. Photomedicine and Laser Surgery. June 2004 22(3): 177-183.

7. Periodontal laser surgery. J Russo : Dent Today (1997) 16:80-1

8. Laser Periodontal therapy ${ }^{\mathrm{TM}}$ Dr.David .A. Pezzullo.

9. Application of Laser in Periodontics: A New Approach in Periodontal Treatment Dr. Dae- hyun Lee, The Hongkong Medical Diary, Vol:12:No:10 Oct 2007.

10. Introduction to Lasers, Functional materials,Sarland Dr. Andrés precautions required for the newer technologies. Improper irradiation of teeth and periodontal pockets by lasers can damage the tooth and root surfaces as well as the attachment apparatus at the bottom of the pocket. Possible damage to the underlying bone and dental pulp should also be considered. ${ }^{9}$

\section{Conclusion :}

In summary, laser treatment is expected to serve as an alternative or adjunctive to conventional mechanical periodontal treatment. Currently, among the different types of lasers available, NdYAG,Er:YAG and Er,Cr:YSGG laser possess characteristics suitable for dental treatment, due to its dual ability to ablate soft and hard tissues with minimal damage. In addition, its bactericidal effect with elimination of lipopolysaccharide, ability to remove bacterial plaque and calculus, irradiation effect limited to an ultra-thin layer of tissue, faster bone and soft tissue repair, make it a promising tool for periodontal treatment including scaling and root surface debridement.

Finally, in order to have a successful periodontal treatment in long term, patients need to be motivated. It is not so much the technology but the motivation and psychology that matter when it comes to practice of oral hygiene before, during and after the periodontal treatment to maintain a good and stable periodontal condition.

Lasagni Lehrstuhl für Funktionswerkstoffe Sommersemester 2007.

11. Lasers in Periodontics - Academy Report JP 2002; 73:1231-1239.

12. Lasers and Light amplification in Dentistry, Use of Lasers in Periodontics. DCNA Vol44,No:40ct. 2000.

13. Lasers in Dentistry. Leo J. Miserendino quintessence Books

14. Lasers in periodontics, position paper; JP 1996; 67; 826-830.

15. Current status of lasers in soft tissue Dental Surgery. Robert M.Pick Journal of Clinical Periodontology 1993; 64:589-602.

16.Laser application in Oral and Maxillofacial surgery-Catone G.A.

17. Treatment of dentine hypersensitivity by lasers, Kimara Y,Wilder-Smith P,Yonaga K: J Clin Periodontology 2000; 27: 715-721.

18. Potential Applications of Erbium:YAG Laser in Periodontics,Ishikawa I,Takasaki AA. Journal of Periodontal Research 2004: 39;275-285.

19. The Impact of Laser Apllication on Periodontal and Peri-implant wound healing, Frank Schwaz,Akira Aoki,Anton Sculean \& Jurgn Becker;Periodontology 2000,Vol 51,2009,79-108.

20. Application of lasers in periodontics:true innovation or myth? Isao Ishikawa, Akira Aoki,Aristeo A,Takasaki, Koji Mizutani,Katia M.Sasaki\& Yuichi Izumi, Periodontology 2000;Vol:50;2009;90-126. 